\title{
Local Optimum Based Power Allocation Approach for Spectrum Sharing in Unlicensed Bands
}

\author{
Furqan Ahmed and Olav Tirkkonen \\ Helsinki University of Technology \\ Espoo, Finland \\ \{furqan.ahmed, olav.tirkkonen\}@tkk.fi
}

\begin{abstract}
We present a novel local optimum based power allocation approach for spectrum sharing in unlicensed frequency bands. The proposed technique is based on the idea of dividing the network in a number of smaller sub-networks or clusters. Sum capacity of each cluster is maximized subject to constraint on total power of each user in a cluster. On its turn each user in a cluster maximizes the sum capacity by calculating power allocations that correspond to a local optimum. Total power constraint of each user and effect of interference from other users in the network is taken into account for finding local optimum solution. Comparison of achieved network sum capacity is done with the well known iterative water filling method. Numerical results show that the proposed cluster based local optimum method achieves higher capacity than selfish iterative water filling and is therefore suitable for geographically distributed networks.
\end{abstract}

\section{Introduction}

Resource allocation for devices working in unlicensed bands has gained significant research interest because of its impact on the performance. An efficient resource allocation is the one in which it is not possible to improve the performance of one system without causing degradation in some other systems performance.

Our focus in this paper is on efficient power allocation for devices in unlicensed bands. We discuss a scenario where a number of users are sharing spectrum in an unlicensed band. The main aim is to find power allocation for each node that maximizes the sum capacity of entire network. Given the importance of this problem, a number of authors have addressed it using different analysis techniques. The well known selfish iterative water filling (IWF) power allocation method was proposed in [1] using a game theoretical approach. In [2] it has been extended and comparison of different power allocation approaches is also given. Most studies have been done considering flat fading case, however flat fading results have been generalized for frequency selective fading channels in [3]. Some other recent works related to distributed power allocation problem include [4], which discusses maximization of a logarithmic utility function or capacity, jointly for all the links. Method discussed by [4] assumes that all the distributed decision makers have information of the price of 
interference that is caused by them to all receivers of the network. Same problem has recently been addressed in [5] for cognitive radio networks.

In this paper we examine the performance of similar power allocation scheme as in [2] but the network model is changed to a more random one. A concept of distributed power allocation is presented for capacity maximization within different clusters. The rest of the paper is organized as follows: in section 2 system model is developed and parameters used throughout the paper are introduced. Section 3 presents the explanation of our proposed local optimum based power allocation scheme. In section 4, performance is analyzed with the help of a numerical example and simulation results are presented.

\section{Network Model}

In this section we describe the system model that includes network architecture, power constraints on transmitter-receiver pairs and expressions for calculating the sum capacity of the network. The network model used in this study is similar to the network examined in [2], but the locations of transmitters and receivers are more random. Figure 1 shows an example layout of the network with 16 links.

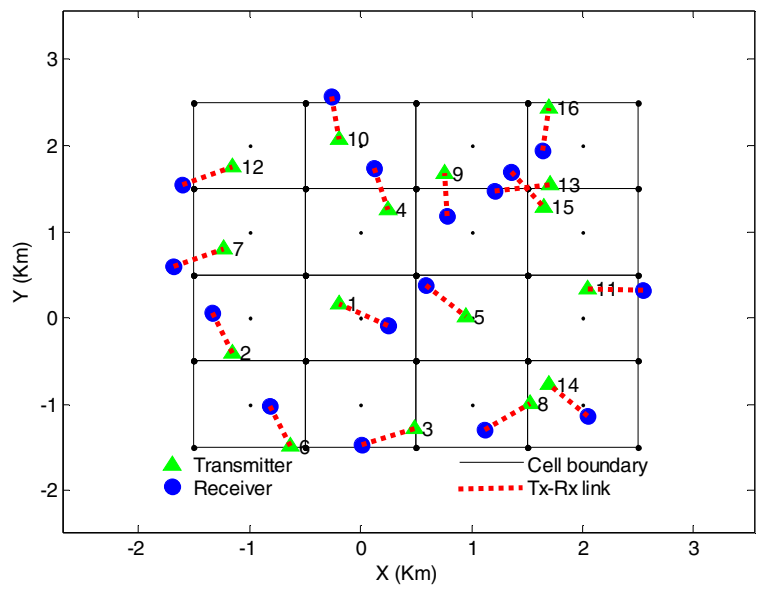

Fig. 1. Network architecture

Network consists of square shaped cells with a transmitter receiver pair (also called link) in each cell. The alignment and directions of links are random with middle point of each link lying randomly in square shaped cell. Numbered triangles indicate the transmitters and circles indicate locations of receivers in network. The links in network share the same frequency band having bandwidth equal to B. Bandwidth is divided into $\mathrm{N}$ number of channels and transmitters of all links can allocate their transmit power freely over these $\mathrm{N}$ channels. All the transmitters have fixed maximum transmit power which they cannot exceed. The Shannon capacity achieved by one link is given by the expression: 


$$
C_{r}=\sum_{j=1}^{N} \log _{2}\left(1+\frac{p_{r, j} g_{r, r, j}}{I_{r, j}+N_{0}}\right) .
$$

Where $N$ is the number of channels, $p_{r, j}$ is the power allocated by link $r$ on channel $j$ and $g_{t, r, j}$ is the gain from transmitter $t$ to receiver on channel $j$. The spectral density of additive white Gaussian noise is $N_{o}$ and $I_{r, j}$ is the interference from other transmitters in network that receiver $r$ experiences on channel $j$. The interference can be obtained as follows:

$$
I_{r, j}=\sum_{\substack{t=1 \\ t \neq r}}^{N} p_{t, j} g_{t, r, j} .
$$

To simplify we define:

$$
\tilde{I_{r, j}}=\frac{I_{r, j}+N_{0}}{g_{r, r, j}} .
$$

Using this notation an equivalent expression for the capacity of link $r$ denoted by $C_{r}$ is given by:

$$
C_{r}=\sum_{j} \log _{2}\left(1+\frac{p_{r, j}}{\tilde{I}_{r, j}}\right) .
$$

The sum capacity of the network is the sum of capacities of all links and can be determined as follows:

$$
C_{\text {sum }}=\sum_{r=1}^{M} C_{r}
$$

Power allocation of link $r$ is defined by vector $p_{r}=\left[p_{r, 1} p_{r, 2} \ldots . . p_{r, N}\right]$ and maximum power constraint that has to be followed by all links in network is characterized as:

$$
\sum_{j=1}^{N} p_{r, j}=P_{\max }
$$

We do not consider the interior points of power constraint equation here. The assumption is that each transmitter uses full power and follows the maximum power constraint. Our aim is to allocate that power efficiently across all channels in an optimum way in order to maximize the network sum capacity.

\section{Local Optimum Based Power Allocation}

In the local optimum based cooperative power allocation scheme, each link is aware of the links within a certain area. The updating link calculates its power to maximize the sum capacity of sub-network and will start using this new allocation. 
To the best of our knowledge, cooperative distributed power allocation based on cluster (as opposed to full network) interference information has not been addressed in literature before. When the cluster becomes large enough to encompass the whole network of all updating links, this scheme is close in spirit to distributed multichannel asynchronous pricing scheme studied in [4]. In [4], the interference to other users is abstracted by a price function but in our case we consider the utility function (capacity) with an aim to directly employ cooperative optimization. In order to optimize power allocation on each link in an asynchronous way, let us consider an updating link $r$ with set of neighbors denoted by $\mu_{\mathrm{r}}$. The index of optimizing link is outside this set i.e. $r \notin \mu_{\mathrm{r}}$. It is assumed that updating link is aware of channel gains between its transmitters and all receivers in cluster as well as interference powers across all channels at all receivers that are included in a cluster. The sum capacity of the cluster is the objective function for optimization and can be expressed as:

$$
C_{\text {sum }, r}=\sum_{j}\left(\log _{2}\left(1+\frac{p_{r, j}}{\tilde{I_{r, j}}}\right)+\sum_{n \in \mu_{r}} \log _{2}\left(1+\frac{f_{n, j}}{p_{r, j}+J_{n, j}}\right)\right)
$$

Where $f_{n j}$ is the scaled transmit power of neighbor $n$ on channel $j$, and $J_{n j}$ is the scaled total interference and noise experienced at channel $j$ of receiver $n$ except the interference caused by $r$. The expressions are given by:

$$
\begin{gathered}
f_{n, j}=p_{n, j} / g_{r, n, j} . \\
J_{n, j}=\tilde{I_{r, j}} / g_{r, n, j}-p_{r, j} .
\end{gathered}
$$

It is assumed that a signaling protocol exists based on which transmitter of link $r$ acquires information of the effect of its transmission to neighbors from which $f_{n, j}$ and $J_{n, j}$ are computed. The objective function is the sum capacity given by equation 7 , and will be optimized under power constraint equation given by 6 . Resulting power allocations will maximize the capacity of cluster. Like iterative water filling approach, the cooperative local optimum needs to be iterated over several asynchronous power allocation updates by all links.

A numerical example of cooperative local optimal power allocation is presented in next section in which we compare the cumulative density functions of the capacities obtained using both techniques. The capacity cumulative density function of cluster based approach is compared with selfish iterative water filling and random power allocation methods. Simulation results show that sum capacity achieved by optimizing cluster capacities is higher than the one achieved by using distributed selfish iterative water filling approach.

\section{Simulation Results}

In this section, we present simulation results to evaluate the performance of proposed scheme. To achieve numerical results, more specific model assumptions have been made. Details of parameters and values used in the simulation are given in table 1. 
Table 1. Simulation parameters

\begin{tabular}{||l|c|c||}
\hline \hline Parameter & Symbol & Value \\
\hline Total bandwidth & $B$ & $10 \mathrm{MHz}$ \\
\hline No. of links & $M$ & 16 \\
\hline No. of channels & $N$ & 4 \\
\hline Max. Tx power & $P_{\max }$ & $16 \mathrm{dBm}$ \\
\hline Thermal noise level & $\mathrm{N}_{\mathrm{T}}$ & $-174 \mathrm{dBm} / \mathrm{Hz}$ \\
\hline Noise figure & $\mathrm{N}_{\mathrm{F}}$ & $6 \mathrm{~dB}$ \\
\hline Path loss exponent & $\alpha$ & 3.76 \\
\hline
\end{tabular}

Figure 2 shows the clusters used in simulations of local optimum approach; the entire network is divided into 4 sub-networks or clusters $\mathrm{L}_{1}, \mathrm{~L}_{2}, \mathrm{~L}_{3}$ and $\mathrm{L}_{4}$. To optimize objective functions under given power constraints we have used built-in MATLAB optimization function called fmincon. Performance is evaluated by comparing capacity cumulative density functions obtained by maximized capacities calculated using different power allocation schemes. Using the simulation parameters specified in table 1, Monte Carlo simulation method is used for calculating capacities for local optimum and selfish iterative water filling schemes. Using the iterative water filling solution as starting point, on its turn, transmitter of a link selected randomly from cluster updates its power allocation to maximize the sum capacity of the cluster on the basis of most recent interference and power situation signaled by neighbors. Randomly ordered optimizations are performed by links of all four sub-networks, selected one by one. We compare the capacity CDF of local optimum based cluster capacity maximization power allocation scheme with selfish iterative water filling method as well as random power allocation. The network sum capacity CDFs are compared in figure 3 .

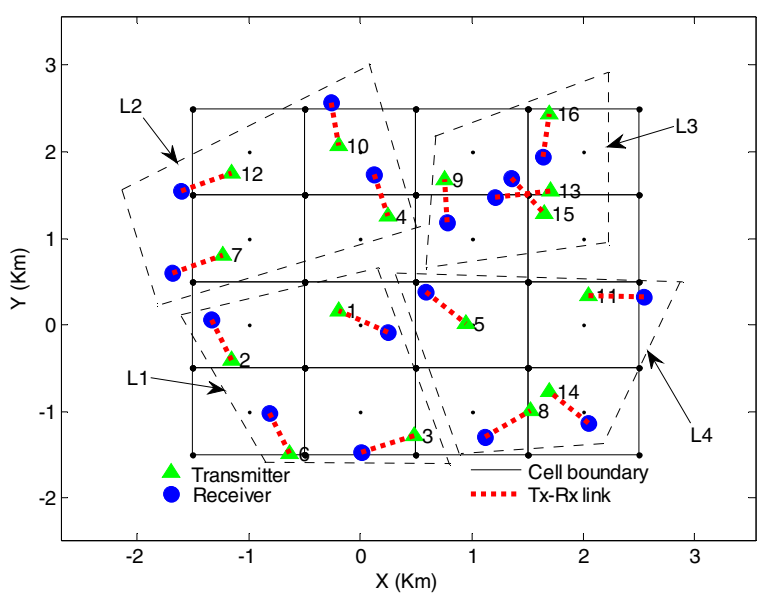

Fig. 2. Network architecture with four clusters 


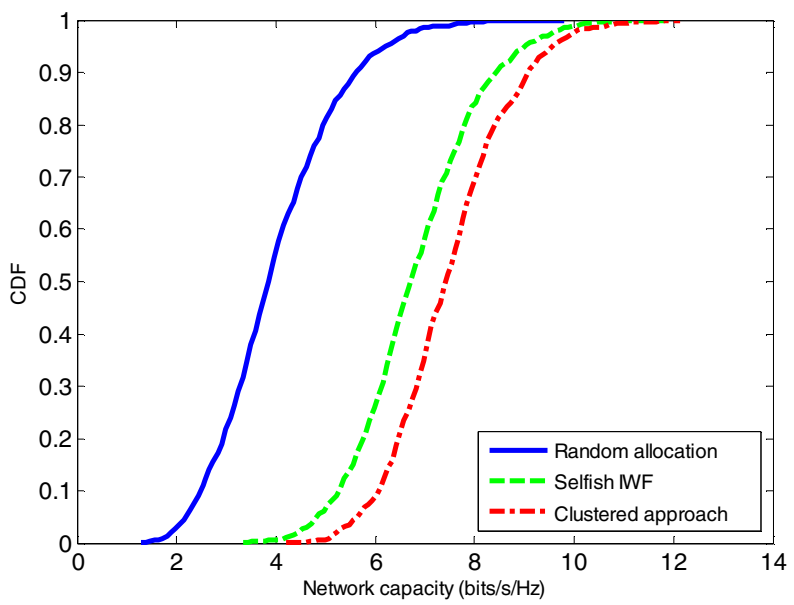

Fig. 3. Comparison of network sum capacity CDFs

Network sum capacity for proposed cooperative cluster based distributed power allocation was found to be around $0.7 \mathrm{bits} / \mathrm{s} / \mathrm{Hz}$ greater than the mean network sum capacity achieved using selfish iterative water filling. We conclude that cluster based locally optimal power allocations is an effective distributive power allocation strategy which can achieve higher network sum capacity than selfish iterative water filling scheme.

\section{References}

1. Chung, S.T., Kim, S.J., Lee, J., Cioffi, J.M.: A Game-Theoretic Approach to Power Allocation in Frequency-Selective Gaussian Interference Channels. In: Proc. IEEE International Symposium on Information Theory, p. 316 (2003)

2. Etkin, R., Parekh, A., Tse, D.: Spectrum Sharing for Unlicensed Bands. IEEE J. Sel. Areas Comm. 25(3), 517-528 (2007)

3. Xu, Y., Chen, W., Cao, Z.: Optimal Power Allocation for Spectrum Sharing in Unlicensed Bands. IEEE Comm. Letters 12(7), 511-513 (2008)

4. Huang, J., Berry, R.A., Honig, M.L.: Distributed Interference Compensation for Wireless Networks. IEEE J. Sel. Areas Comm. 24(5), 1074-1084 (2006)

5. Lee, W.Y., Akyildiz, I.F.: Joint Spectrum and Power Allocation for Inter-Cell Spectrum Sharing in Cognitive Radio Networks. In: Proc. DySPAN, October 2008, pp. 1-12 (2008) 